\title{
Avaliação da rugosidade superficial de três resinas compostas submetidas a diferentes técnicas de polimento ${ }^{\dagger}$
}

\section{Evaluation of the superficial roughness of three composite resins submitted to different polishing techniques}

\author{
Benícia Carolina Iaskieviscz RIBEIRO* \\ Margareth ODA** \\ Edmir MATSON***
}

\begin{abstract}
RIBEIRO, B. C. I.; ODA, M.; MATSON, E. Avaliação da rugosidade superficial de três resinas compostas submetidas a diferentes técnicas de polimento. Pesqui Odontol Bras, v. 15, n. 3, p. 252-256, jul./set. 2001.

Neste estudo, avaliou-se a rugosidade superficial de resinas classificadas como compactáveis e uma híbrida tradicional comparando-se diferentes técnicas de polimento. Confeccionaram-se corpos-de-prova, que foram armazenados em água destilada por 24 horas e posteriormente submetidos a duas técnicas de tratamento superficial, em que foram empregados dois sistemas de discos de acabamento e polimento e pontas siliconizadas. Os resultados revelaram maior rugosidade superficial da resina Alert ${ }^{\mathrm{TM}}$ em relação a Solitaire ${ }^{\circledR}$ e Degufill Mineral ${ }^{\circledR}$, não havendo diferenças estatisticamente significantes entre as últimas citadas. A rugosidade superficial das restaurações cujo polimento foi executado com as pontas Enhance ${ }^{\circledR}$ foi maior que o polimento obtido com os sistemas de discos, não havendo diferenças estatisticamente significante entre esses.
\end{abstract}

UNITERMOS: Resinas compostas; Polimento dentário.

\section{INTRODUÇÃO}

A hipervalorização da estética aliada à evolução das resinas compostas e a introdução de novos produtos têm proporcionado uma maior indicação das mesmas para dentes posteriores.

Há muita diversificação de técnicas de acabamento e polimento ${ }^{7,15}$ e polemiza-se que a rugosidade superficial independe do tamanho das partículas. Inúmeros autores ${ }^{2,7,12,13}$ consideram relevante $o$ intervalo de tempo entre o término da restauração e o polimento. No entanto, existem considerações contrárias que recomendam o polimento imediato após a polimerização ${ }^{3}$.

A remoção de excessos de uma restauração é denominada redução inicial, acabamento quando se obtêm partículas maiores que $25 \mu \mathrm{m}$ e polimento quando menores que $25 \mu \mathrm{m}^{5}$. Lisura define a análise de uma superfície polida e tem por objetivo medir o polimento ${ }^{6}$. Este reporta-se a refinamento, lisura superficial, lustro ou brilho e o resultado fi- nal de todos esses termos implica em reflectância de luz ${ }^{1}$.

O surgimento de um novo material indicado para fabricação de resinas compostas posteriores compactáveis foi citado em 1997 e o tratamento superficial deste obtém-se com pontas para compósitos destinadas para esta finalidade ${ }^{9,10}$. Utilizando técnicas de polimento convencionais para compósitos posteriores, LEINFELDER et al. ${ }^{11}$ (1998b) obtiveram a mesma lisura.

Considerando-se a introdução de resinas classificadas como compactáveis, procurou-se, neste estudo, avaliar a rugosidade superficial das mesmas em relação à uma resina híbrida e comparar o efeito de diferentes técnicas de polimento.

\section{MATERIAIS E MÉTODOS}

Foram selecionadas três resinas compostas indicadas para restaurações diretas em dentes posteriores (Solitaire ${ }^{\circledR}$, Alert ${ }^{\mathrm{TM}}$ e Degufill Mineral ${ }^{\circledR}$ ) com as quais confeccionaram-se os corpos-de-prova.

\footnotetext{
† Resumo da Dissertação de Mestrado, Área de Concentração em Dentística - Faculdade de Odontologia da USP em convênio interinstitucional com a Universidade Federal do Mato Grosso do Sul.

* Professora Assistente da Universidade Federal do Mato Grosso do Sul.

** Professora Doutora; ***Professor Titular - Departamento de Dentística, Faculdade de Odontologia da USP.
} 
RIBEIRO, B. C. I.; ODA, M.; MATSON, E. Avaliação da rugosidade superficial de três resinas compostas submetidas a diferentes técnicas de polimento. Pesqui Odontol Bras, v. 15, n. 3, p. 252-256, jul./set. 2001.

Utilizaram-se também três materiais para as técnicas de polimento, sendo dois sistemas de discos (Sof-Lex ${ }^{\mathrm{TM}}$ e Super-Snap ${ }^{\circledR}$ ) e pontas siliconizadas (Enhance ${ }^{\circledR}$ ) (Quadros 1 e 2).

Para a confecção dos corpos-de-prova utilizou-se um dispositivo desenvolvido por NAVARRO et al. ${ }^{14}$ (1998) de estrutura metálica com uma projeção de $4 \mathrm{~mm}$ de altura por $6 \mathrm{~mm}$ de diâmetro. Este foi moldado e, na área correspondente à projeção, construíram-se os corpos-de-prova em resina composta. Utilizaram-se três incrementos, com espessura média de $1,5 \mathrm{~mm}$ cada um, que foram polimerizados por 40 segundos, com aparelho de luz (Curing Light XL 1500 - 3M) obtendo-se, ao final, uma pastilha de resina composta de $4,0 \mathrm{~mm}$ de altura por $6,0 \mathrm{~mm}$ de diâmetro. Removida do molde, a parte que esteve em contato com o fundo deste era submetida a mais uma polimerização, totalizando 160 segundos de exposição à luz visivel.

A distribuição dos corpos-de-prova, em grupos de dez, foi realizada de acordo com a marca comercial (Solitaire ${ }^{\circledR}$, Alert ${ }^{\mathrm{TM}}$ e Degufill Mineral ${ }^{\circledR}$ ). Como estes seriam submetidos a três materiais de polimento, confeccionaram-se trinta corpos de cada resina composta.

Estes foram fixados em nove placas de vidro (dez corpos por placa e três placas por resina) com o adesivo instantâneo Super Bonder (Loctite Brasil Ltda.) num total de noventa corpos-de-prova. Foram armazenados em água destilada por 24 horas a temperatura ambiente e, após esse periodo, submetidos às técnicas de polimento e posterior leitura da sua superficie.

QUADRO 1 - Resinas compostas testadas.

\begin{tabular}{||l|l|l||}
\hline Marca comercial & \multicolumn{1}{|c||}{ Característica } & \multicolumn{1}{|c|}{ Fabricante } \\
\hline Solitaire & $\begin{array}{l}\text { "Polyglass” foto- } \\
\text { polimerizável }\end{array}$ & $\begin{array}{l}\text { Heraus Kulzer } \\
\text { Gmbh }\end{array}$ \\
\hline Alert & $\begin{array}{l}\text { Resina composta } \\
\text { condensável, ma- } \\
\text { triz fibrosa }\end{array}$ & $\begin{array}{l}\text { Jeneric/Pentron } \\
\text { Incorporated }\end{array}$ \\
\hline Degufill Mineral $^{\circledast}$ & Resina híbrida & Degussa S. A. \\
\hline
\end{tabular}

O primeiro grupo (resina Solitaire) foi polido com discos da marca Sof-Lex ${ }^{\mathrm{TM}}$ (dorso azul-escuro, azul médio e azul-claro), diâmetro de 19,05 mm, de forma intermitente, em rotação convencional e decorridos trinta segundos o mesmo era descartado. O corpo-de-prova era lavado com "spray" para remover debris, seco com jato de ar e, em seguida, submetido a outro disco de granulometria menor.

Igual procedimento foi executado com os discos Super Snap ${ }^{\circledR}$ empregados seqüencialmente, da granulometria maior para a menor, por 30 segundos, em baixa velocidade.

As pontas Enhance foram utilizadas também em velocidade convencional, por 30 segundos, e após esse periodo descartadas.

O mesmo procedimento e as mesmas técnicas de polimento foram aplicadas para os grupos constituídos das resinas Alert ${ }^{\mathrm{TM}}$ e Degufill Mineral ${ }^{\circledR}$.

A leitura do polimento executado nos corpos-de-prova foi realizada com o aparelho Surftest 211, série 178, da marca Mitutoyo. Este possui uma ponta apalpadora com tamanho de 0,005 $\mathrm{mm}$ de raio que se desloca à uma velocidade de $0,5 \mathrm{~mm} / \mathrm{s}$, conectada a um visor de cristal líquido que registra digitalmente a leitura da superficie. Foi regulado para percorrer uma distância de 0,25 mm, escala em micrômetros e ajuste em $\mathrm{Ra}$, sendo esta a média aritmética encontrada entre picos e vales das superfícies submetidas à análise. Esta prévia calibragem foi realizada sobre uma superficie padrão pertencente ao próprio aparelho.

Para cada grupo, realizaram-se quatro leituras por corpo-de-prova, totalizando quarenta medidas por técnica de polimento empregada e cento e vinte para cada tipo de resina composta, resultando trezentos e sessenta medidas em todo o experimento.

\section{RESULTADOS E DISCUSSÃO}

Objetivando identificar se existem diferenças estatisticamente significantes ou não, entre as resinas estudadas e entre os polimentos, aplicou-se o teste análise de variância observado na Tabela 1 .

Pela análise desta, verifica-se que houve diferenças estatisticamente significantes entre as mé-

QUADRO 2 - Materiais de polimento testados.

\begin{tabular}{|c|c|c|}
\hline Marca comercial & Características & Fabricante \\
\hline Sof-Lex ${ }^{\mathrm{TM}}$ & Óxido de alumínio & 3M do Brasil Ltda. \\
\hline Super Snap ${ }^{\circledR}$ & Poliéster Mylar da Dupont, Base de silicone & Shofu Dental Inc. \\
\hline Enhance $^{\circledR}$ & $\begin{array}{l}\text { Tripolímero (estireno-butadieno-metacrilato de metila), sílica pirolítica silanizada, } \\
\text { uretano dimetacrilato, canforoquinona n-metil dietanolamina, óxido de alumínio }\end{array}$ & $\begin{array}{l}\text { Dentsply Indústria e } \\
\text { Comércio Ltda. }\end{array}$ \\
\hline
\end{tabular}


RIBEIRO, B. C. I.; ODA, M.; MATSON, E. Avaliação da rugosidade superficial de três resinas compostas submetidas a diferentes técnicas de polimento. Pesqui Odontol Bras, v. 15, n. 3, p. 252-256, jul./set. 2001.

dias obtidas em relação a rugosidade superficial das resinas compostas e o tipo de polimento. Entretanto, para saber qual ou quais médias diferem entre si, aplicou-se o teste de Tukey ao nível de 0,01 de significância.

Pela análise da Tabela 2, verifica-se que a resina Alert ${ }^{\mathrm{TM}}$ apresentou maior rugosidade superficial em relação ao produto Solitaire ${ }^{\circledR}$ sendo estatisticamente significante essa diferença. Observaram-se entre as médias obtidas das resinas Degufill Mine$\mathrm{ral}^{\circledR}$ e Alert ${ }^{\mathrm{TM}}$ diferenças estatisticamente significantes, sendo que a primeira apresenta maior lisura. Porém, entre as resinas Solitaire ${ }^{\circledR}$ e Degufill Mineral $^{\circledR}$ não se observaram diferenças estatisticamente significantes, como pode ser visualizado no Gráfico 1.

Estes resultados podem ser explicados devido a distinção entre os constituintes orgânicos e inorgânicos dos compósitos estudados. As resinas compactáveis apresentam partículas mais grossas e texturizadas que outros sistemas ${ }^{10}$, podendo se apresentar em forma de fibras, partículas porosas ou irregulares ${ }^{16}$. A diferença encontrada entre as resinas Solitaire ${ }^{\circledR}$ e Alert ${ }^{\mathrm{TM}}$ era esperada desde o início quando detectamos dissensões entre elas, ha-

TABELA 1 - Análise de variância para as médias dos valores da rugosidade superficial das resinas compostas tratadas com diferentes técnicas de polimento e teste $\mathrm{F}$ ao nível de $99 \%$ de confiança.

\begin{tabular}{l|c|c|c|c}
\hline \hline $\begin{array}{l}\text { Fonte de } \\
\text { variação }\end{array}$ & $\begin{array}{c}\text { Quadrados } \\
\text { médios }\end{array}$ & $\begin{array}{c}\text { Graus de } \\
\text { liberdade }\end{array}$ & $\begin{array}{c}\text { Quadrado } \\
\text { médio do } \\
\text { resíduo }\end{array}$ & $\mathrm{F}$ \\
\hline Resina & 0,0750 & 2 & 0,0060 & $12,48^{*}$ \\
\hline Polimento & 0,8830 & 2 & 0,0064 & $132,94^{*}$ \\
\hline $\begin{array}{l}\text { Resina } \\
\text { versus po- } \\
\text { limento }\end{array}$ & 0,0033 & 4 & 0,0064 & 0,5156 \\
\hline \hline
\end{tabular}

*Significante ao nível de 0,01.

TABELA 2 - Médias dos resultados da rugosidade superficial das resinas compostas e a probabilidade obtida pelo teste de Tukey ao nivel de 0,01 de significância.

\begin{tabular}{l|l|l|l}
\hline \hline \multicolumn{1}{c|}{ Resina } & Solitaire $^{\circledR}$ & \multicolumn{1}{|c|}{ Alert $^{\mathrm{TM}}$} & \multicolumn{1}{c}{$\begin{array}{c}\text { Degufill } \\
\text { Mineral }^{\circledR}\end{array}$} \\
\hline Médias & 0,1649 & 0,2641 & 0,2035 \\
\hline Solitaire $^{\circledR}$ & & $0,000213^{*}$ & 0,149503 \\
\hline Alert $^{\mathrm{TM}}$ & $0,000213^{*}$ & & $0,014534^{*}$ \\
\hline Degufill Mineral $^{\circledR}$ & 0,149503 & $0,014534^{*}$ & \\
\hline \hline
\end{tabular}

*Significante ao nível de 0,01. vendo uma maior facilidade de manipulação, inserção e compactação da resina Solitaire ${ }^{\circledR}$, assim como menor viscosidade e maior lisura superficial, mesmo antes da polimerização deste compósito em relação à resina Alert ${ }^{\mathrm{TM}}$. Esta apresenta uma textura superficial mais áspera, dilacera-se no momento da inserção, possui aspecto seco e opaco, o oposto do recomendado ${ }^{6}$ - que as resinas compostas devam parecer úmidas e brilhantes. Concordamos com os autore ${ }^{16}$ que consideram dificil o acabamento e polimento da resina Alert ${ }^{\mathrm{TM}}$, porém adequado o da resina Solitaire ${ }^{\circledR}$. Os resultados demonstraram ainda, não haver diferença estatisticamente significante entre as resinas Solitaire ${ }^{\circledR}$ e Degufill Mineral ${ }^{\circledR}$.

Na Tabela 3, observam-se as médias dos resultados da rugosidade superficial dos diferentes tipos de polimento e a probabilidade obtida pelo teste de Tukey, ao nível de 0,01 de significância. Pode-se verificar que existem diferenças estatisticamente significantes entre as médias da rugosidade superficial nos polimentos executados com os sistemas de discos e as pontas siliconizadas. Notam-se superficies inquestionavelmente mais lisas quando tratadas com os discos e mais rugosas

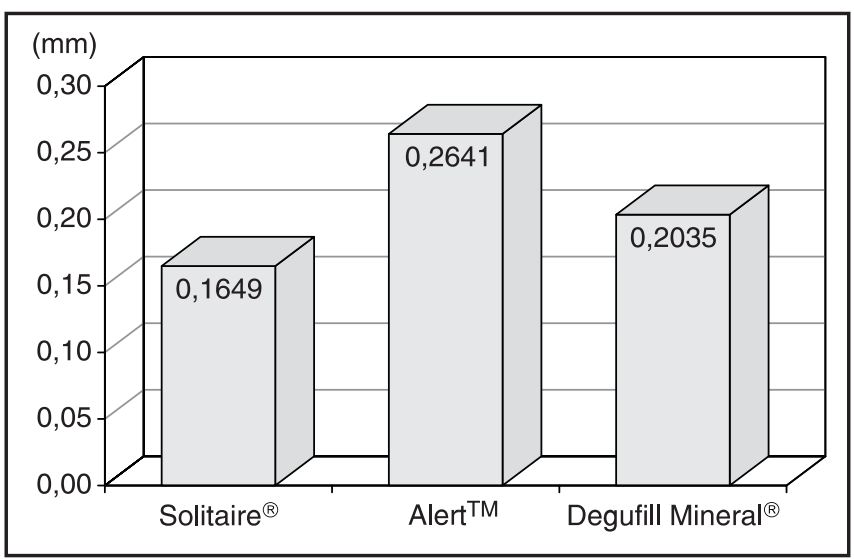

GRÁFICO 1 - Médias da rugosidade superficial das resinas compostas.

TABELA 3 - Médias de rugosidade superficial dos diferentes tipos de polimento e a probabilidade obtida pelo teste de Tukey.

\begin{tabular}{l|c|c|c}
\hline \hline \multicolumn{1}{c|}{ Polimento } & Sof-Lex $^{\mathrm{TM}}$ & Super Snap $^{\circledR}$ & Enhance $^{\circledR}$ \\
\hline Médias & 0,112250 & 0,1114167 & 0,40900 \\
\hline Sof-Lex & & 0,999220 & $0,000121^{\text {TM }}$ \\
\hline Super-Snap $^{\circledR}$ & 0,999220 & & $0,000121^{*}$ \\
\hline Enhance $^{\circledR}$ & $0,000121^{*}$ & $0,000121^{*}$ & \\
\hline \hline
\end{tabular}

*Significante ao nível de 0,01. 
RIBEIRO, B. C. I.; ODA, M.; MATSON, E. Avaliação da rugosidade superficial de três resinas compostas submetidas a diferentes técnicas de polimento. Pesqui Odontol Bras, v. 15, n. 3, p. 252-256, jul./set. 2001.

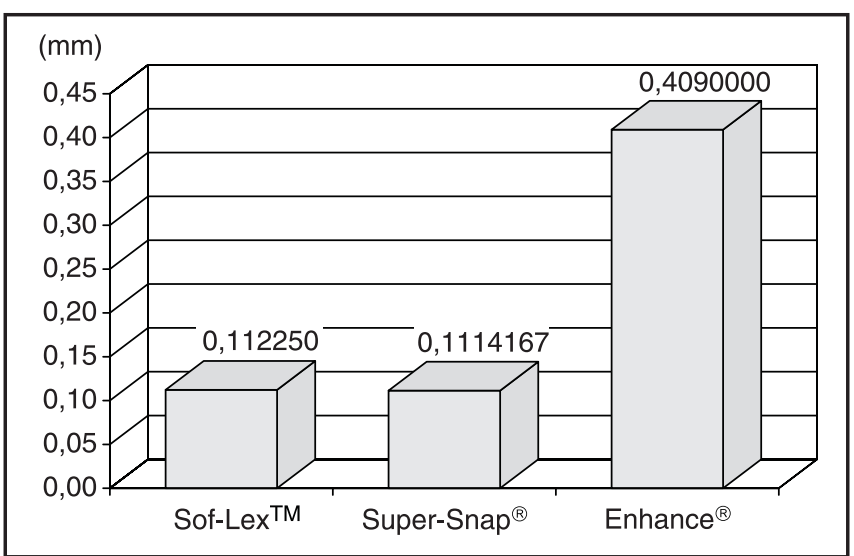

GRÁFICO 2 - Médias da rugosidade superficial dos diferentes tipos de polimento.

quando usadas as pontas siliconizadas. Estes dados podem ser observados no Gráfico 2 .

Resultado semelhante foi obtido por SETCOS et al. ${ }^{18}$ (1999) com esses mesmos materiais, porém, empregados sobre a superficie de resinas compostas híbridas e microparticuladas. Autores como SANTOS et al. ${ }^{17}$ (1984), TAYRA et al. ${ }^{19}$ (1985) e ou$\operatorname{tros}^{6}$ observaram melhor desempenho no tratamento superficial de resinas compostas com discos de óxido de alumínio. No entanto, o uso de pontas de borracha Enhance ${ }^{\circledast}$ tanto para contorno como polimento de áreas posteriores e oclusais é defendido por MONDELLI ${ }^{13}$ (1995), SETCOS et al. ${ }^{19}$ e outros ${ }^{6,16}$ que as consideram clinicamente aceitáveis, assim como os discos Super-Snap ${ }^{\circledR}$ para áreas de difícil alcance ${ }^{6}$.

BARATIERI et al. ${ }^{2}$ (1995) e LOGUERCIO ${ }^{12}$ (1997) observaram que o polimento proporciona maior tolerância dos tecidos periodontais às restaurações e de acordo com CHUNG ${ }^{4}$ (1994) estes procedimentos geram uma diminuição na rugosidade, variando de 26 a $74 \%$.

\section{CONCLUSÕES}

No teste de rugosimetria, a resina composta compactável que possui matriz fibrosa apresentou maior rugosidade superficial em relação aos outros compósitos estudados, sendo essa diferença estatisticamente significante.

Entre as outras resinas compostas, uma híbrida e a outra compactável, não foram detectadas diferenças estatisticamente significantes entre si em relação à rugosidade superficial.

As técnicas de polimento apresentaram diferenças estatisticamente significantes, sendo que os sistemas de acabamento e polimento que utilizam discos não foram diferentes significantemente entre si.

Entretanto, as pontas siliconizadas apresentaram maior rugosidade em relação aos sistemas de discos, sendo essa diferença inferior estatisticamente.

RIBEIRO, B. C. I.; ODA, M.; MATSON, E. Evaluation of the superficial roughness of three composite resins submitted to different polishing techniques. Pesqui Odontol Bras, v. 15, n. 3, p. 252-256, jul./set. 2001.

In this study, the superficial roughness of two composite resins classified as condensable and of a traditional hybrid composite resin was analyzed after different polishing techniques. Test specimens were confected, immersed in distilled water for 24 hours and submitted to two different techniques of superficial treatment, in which disc systems $\left(\right.$ Sof-Lex ${ }^{\mathrm{TM}}$ and Super Snap ${ }^{\mathbb{B}}$ ) and silicon tips (Enhance ${ }^{\mathbb{B}}$ ) were utilized. The results revealed the greater superficial roughness of Alert ${ }^{\mathrm{TM}}$, when compared with Solitaire ${ }^{\circledR}$ and Degufill Mineral ${ }^{\circledR}$ - no statistically significant differences were observed between the last two materials. The superficial roughness of the specimens polished with Enhance ${ }^{\circledR}$ tips was greater than that of the specimens polished with discs and there were no statistically significant differences between both disc systems.

UNITERMS: Composite resins; Dental polishing.

\section{REFERÊNCIAS BIBLIOGRÁFICAS}

1. ACABAmEnTO e polimento de resinas compostas. Dent Advisor, v. 5, n. 4, p. 1-5, maio 1998.

2. BARATIERI, L. N.; MONTEIRO Jr., S.; ANDRADA, M. A. C. et al. Estética: restaurações adesivas diretas em dentes anteriores fraturados. São Paulo: Santos, 1995a. Cap. 7. p. 209-261.

3. BRACKETT, W. W.; GILPATRICK, R. O.; GUNNING, T. D. Effect of the finishing method on the microleakage of class V resin composite restorations. Am J Dent, v. 10, n. 4, p. 181-191, Aug. 1997.

4. DODGE, W. W.; DALE, R. A.; COOLEY, R. L.; DUKE, E. S. Comparison of wet and dry finishing of resin composites with aluminum oxide discs. Dent Mater, v. 7, n. 1, p. 18-20, Jan. 1991.

5. FINISHING and polishing. Dent Advisor, v. 5, n. 3, p. 1-6, Sept. 1988.

6. FINISHING direct restorations. Adept Report, v. 3, n. 1, p. 1-16, 1992. 
RIBEIRO, B. C. I.; ODA, M.; MATSON, E. Avaliação da rugosidade superficial de três resinas compostas submetidas a diferentes técnicas de polimento. Pesqui Odontol Bras, v. 15, n. 3, p. 252-256, jul./set. 2001.

7. HERRGOTT, A. M. L.; ZIEMIECKI, T. L.; DENNISON, J. B. An evaluation of different composite resin systems finished with various abrasives. J Am Dent Assoc, v. 119, n. 6, p. 729-732, Dec. 1989.

8. HOELSCHER, D. C.; NEME, A. M. L.; PINK, F. E.; HUGHES, P. J. The effect of three finishing systems on four esthetic restorative materials. Oper Dent, v. 23, n. 1, p. 36-42, Jan./Feb. 1998.

9. LEINFELDER, K. F. New developments in resin restorative systems. J Am Dent Assoc, v. 128, n. 5, p. 573-581, May 1997.

10. LEINFELDER, K. F.; RADZ, G. M.; NASH, R. W. A report on a new condensable composite resin. Compen Contin Educ Dent, v. 19, n. 3, p. 230-237, Mar. 1998a.

11. LEINFELDER, K. F.; LYLES, M. B.; RITSCO, R. G. Amalgam substitute - a new polymer rigid matrix material. Oral Health, v. 88, n. 4, p. 57-62, Apr. 1998b.

12. LOGUÉRCIO, A. D. Acabamento e polimento em restaurações estéticas. In: BUSATO, A. L. S. Dentística: restaurações em dentes anteriores. São Paulo : Artes Médicas, 1997. Cap. 19. p. 368-443.

13. MONDELLI, R. F. L. Uso clínico das resinas compostas em dentes posteriores. Maxi-Odonto Dent, v. 1, n. 3, p. 39-44, maio/jun. 1995.

14. NAVARRO, R. S.; ESTEVES, G.; OLIVEIRA JUNIOR, W. T. et al. Influence of surface treatment, age and type of amalgam alloy - rugosimetric and SEM evaluation. $\mathbf{J}$ Dent Res, v. 77 , n. 5, p. 114 , 1998. [Resumo IADR n. 22].

15. RESINAS compostas anteriores e posteriores. Dent Advisor, v. 1, n. 1, p. 3-6, jan. 1994.

16. RESINAS compostas condensáveis. Dent Advisor, v. 5, n. 8, p. 1-12, set. 1998 .

17. SANTOS, A.; VINHA, D.; PANZERI, H. Restaurações com resina composta - comportamento em função do tratamento superficial e da ação da saliva. RGO, v. 32, n. 1, p. 13-16, jan./mar. 1984.

18. SETCOS, J. C.; TARIM, B.; SUZUKI, S. Surface finish produced on resin composites by polishing systems. Quintessence Int, v. 30, n. 3, p. 169-173, Mar. 1999.

19. TAYRA, M.; GABRIELLI, F.; REIS, M. S. M. C. G. et al. Efeito do acabamento, material e tempo sobre a superficie da resina composta. RGO, v. 23, n. 2, p. 143-150, abr./jun. 1985.

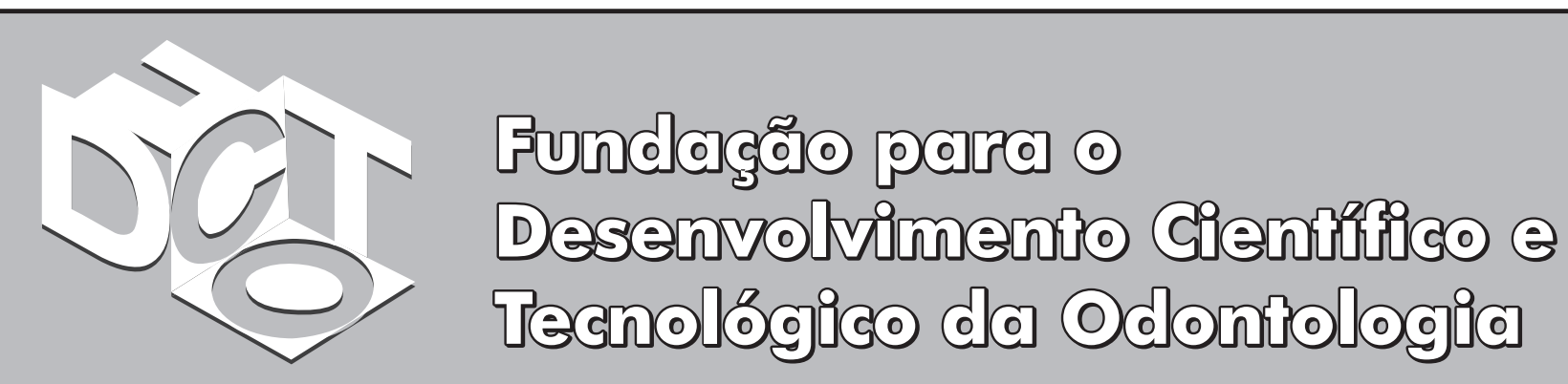

CURSOS PARA O $1^{\circ}$ SEMESTRE DE 2002

Visite-nos no endereço:

Av. Prof. Lineu Prestes, 2227

Cid. Universitária - São Paulo-SP

ESPECIALIZAÇÃO

CEP: 05508-900

ou no site:

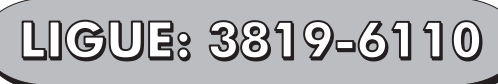

ATUALIZAÇÃO

CURSOS TÉCNICOS

CURSOS EXTRAS

www.fundecto.com.br

WORKSHOPS 